\title{
Proper Motions and the Distance to a Water Vapor Maser in the Galaxy M33
}

\author{
A. L. Argon, L. J. Greenhill, J. M. Moran, \& M. J. Reid \\ Harvard-Smithsonian Center for Astrophysics, Cambridge, MA, U.S.A. \\ K. M. Menten \\ Max-Planck-Institut für Radioastronomie, Bonn, Germany
}

Abstract. The relative proper motions of water vapor maser features in the H II region IC 133 have been used to determine a distance to the galaxy M33 that is independent of the usual calibrations associated with extragalactic distance estimates, e.g., extinction and metallicity.

\section{Observations and Data Reduction}

We observed IC 133 seven times over a 6.3-year period, starting in 1987 June. The antennas used for these observations were: the MPIfR $100 \mathrm{~m}$ (epochs 1-7), the Haystack $37 \mathrm{~m}$ (epochs 1-5), the NRAO $43 \mathrm{~m}$ (epochs 1-5), the VLA phased array (epochs 1-7), the OVRO $40 \mathrm{~m}$ or $25 \mathrm{~m}$ (epochs 1-5), and the VLBA (epochs 6-7). We correlated, calibrated, and imaged the data using standard spectral-line VLBI techniques. Maps from the first two epochs are presented by Greenhill et al. $(1990,1993)$.

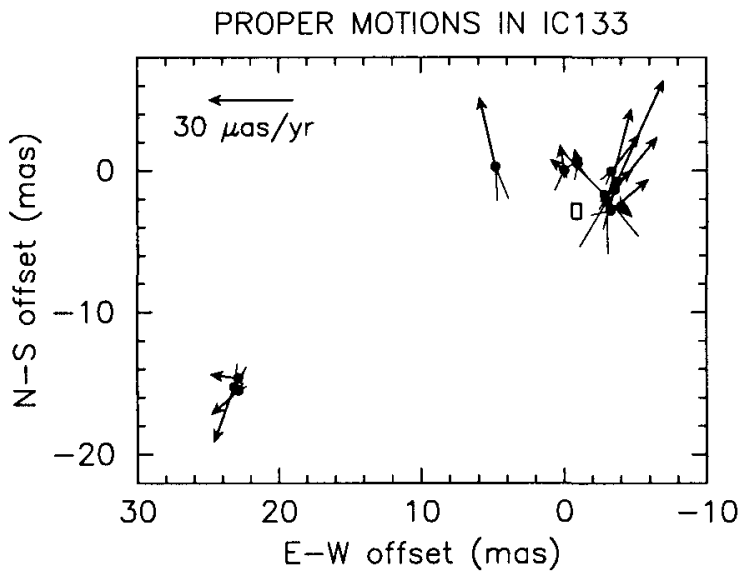

Figure 1. We detected 79 isolated features over a 6.3 -year period and the 14 that persisted for at least three epochs are shown here. The $1 \sigma$ uncertainty in the magnitude and direction of each vector is reflected by an "error cone" on the vector's tail. The center of expansion is marked by a $1 \sigma$ error box. An angular motion of 30 $\mu$ as $\mathrm{yr}^{-1}$ corresponds to a linear motion of $100 \mathrm{~km} \mathrm{~s}^{-1}$ at the nominal distance of $700 \mathrm{kpc}$. A second center of maser activity (IC 133 West), located $\sim 0.3^{\prime \prime}$ from the reference feature, is not shown. 


\section{Distance to $\mathrm{M} 33$}

We model the maser as a uniform outflow from a central source (see Figure 1). This involves solving for seven global parameters and the positions of the maser features along the line of sight (Reid et al. 1988). The distance to the source is that value which best (in a least squares sense) relates the observed angular motions to the model fit linear velocities.

The radial velocity of the peak of the maser emission in IC 133 is at -210 $\mathrm{km} \mathrm{s}^{-1}$ and has been so since its discovery in 1976 (Churchwell et al. 1977). We take this to be the radial velocity of the center of expansion for the following reasons:

- The measured systematic H I velocity for the IC 133 region is $-210 \pm 4$ $\mathrm{km} \mathrm{s}^{-1}$ (Rogstad, Wright, \& Lockhart 1976-angular resolution $2^{\prime}$ ).

- Several independent $H_{\alpha}$ studies suggest a mean radial velocity of $\sim-210$ $\mathrm{km} \mathrm{s}^{-1}$ (de Vaucouleurs \& de Vaucouleurs 1971; Brandt 1965; Mayall \& Aller 1942).

- The radial velocities of the strongest peaks of the maser emission in various sources are often within a few $\mathrm{km} \mathrm{s}^{-1}$ of the maser cloud's mean radial velocity (e.g., Walker, Matsakis, \& García-Barreto 1982; Gwinn, Moran, \& Reid 1992-W49N).

Holding the central LSR velocity fixed at $-210 \mathrm{~km} \mathrm{~s}^{-1}$ and solving for the remaining six global parameters and the line of sight positions, we obtain a distance of $830 \pm 250 \mathrm{kpc}$. The central LSR velocity must be constrained rather than fit because it is highly correlated with distance. Separate solutions at -215 and $-205 \mathrm{~km} \mathrm{~s}^{-1}$ yield distance estimates that are compatible with our quoted error. We emphasize that this is a preliminary distance estimate.

Acknowledgments. The National Radio Astronomy Observatory is a facility of the National Science Foundation, operated under a cooperative agreement by Associated Universities, Inc.

\section{References}

Brandt, J. C. 1965. MNRAS, 129, 309-319.

Churchwell, E., et al. 1977. A\&A, 54, 969-971.

de Vaucouleurs, G., \& de Vaucouleurs, A. 1971. Astrophysical Letters, 8, 17-20.

Greenhill, L. J., et al. 1990. ApJ, 364, 513-526.

Greenhill, L. J., et al. 1993. ApJ, 406, 482-488.

Gwinn, C. R., Moran, J. M., \& Reid, M. J. 1992. ApJ, 393, 149-164.

Mayall, N. U., \& Aller, L. H. 1942. ApJ, 95, 5-23.

Reid, M. J., et al. 1988. ApJ, 330, 809-816.

Rogstad, D. H., Wright, M. C. H., \& Lockhart, I. A. 1976. ApJ, 204, 703-716.

Walker, R. C., Matsakis, D. N., \& García-Barreto, J. A. 1982. ApJ, 255, 128-142. 